Case Report

\title{
Hyperammonemia: What Urea-lly Need to Know: Case Report of Severe Noncirrhotic Hyperammonemic Encephalopathy and Review of the Literature
}

\author{
Ruby Upadhyay, ${ }^{1}$ Thomas P. Bleck, ${ }^{2}$ and Katharina M. Busl ${ }^{3}$ \\ ${ }^{1}$ Department of Neurological Sciences, Rush University Medical Center, 1725 West Harrison Street, POB Suite 1121, \\ Chicago, IL 60612, USA \\ ${ }^{2}$ Rush Medical College, Rush University Medical Center, 600 S. Paulina Street, Chicago, IL 60612, USA \\ ${ }^{3}$ Department of Neurology, Division of Neurocritical Care, University of Florida, McKnight Brain Institute, \\ Room L3-100, 1149 Newell Drive, Gainesville, FL 32611, USA \\ Correspondence should be addressed to Katharina M. Busl; katharina.busl@neurology.ufl.edu
}

Received 13 July 2016; Accepted 28 August 2016

Academic Editor: Piotr K. Janicki

Copyright (C) 2016 Ruby Upadhyay et al. This is an open access article distributed under the Creative Commons Attribution License, which permits unrestricted use, distribution, and reproduction in any medium, provided the original work is properly cited.

Purpose. A 66-year-old man who presented with coma was found to have isolated severe hyperammonemia and diagnosed with a late-onset urea-cycle disorder. He was treated successfully and had full recovery. Methods. We report a novel case of noncirrhotic hyperammonemia and review the literature on this topic. Selected literature for review included English-language articles concerning hyperammonemia using the search terms "hyperammonemic encephalopathy", "non-cirrhotic encephalopathy", "hepatic encephalopathy", "urea-cycle disorders", "ornithine transcarbamylase (OTC) deficiency", and "fulminant hepatic failure". Results. A unique case of isolated hyperammonemia diagnosed as late-onset OTC deficiency is presented. Existing evidence about hyperammonemia is organized to address pathophysiology, clinical presentation, diagnosis, and treatment. The case report is discussed in context of the reviewed literature. Conclusion. Late-onset OTC deficiency presenting with severe hyperammonemic encephalopathy and extensive imaging correlate can be fully reversible if recognized promptly and treated aggressively.

\section{Background}

Ammonia is a highly potent neurotoxin well known for its implication in hepatic encephalopathy [1]. Any case of acute altered mental status should prompt a consideration of hyperammonemia as a potential cause. When hyperammonemia is detected, the etiology of its accumulation must be determined in order to guide treatment. While hyperammonemia in adults is related to severe, often cirrhotic, liver disease in $90 \%$ of cases [2], increased ammonia production or decreased ammonia elimination is one of the alternative etiologies of hyperammonemia [1]. Awareness of hyperammonemia in absence of severe hepatic disease may lead to lifesaving prompt diagnosis and treatment.

We present a case of acute severe noncirrhotic hyperammonemia and review its pathophysiology, diagnostic, and treatment considerations.

\section{Case Presentation}

A 66-year-old male business manager with past medical history of hypertension, diabetes mellitus, and intermittent sinusitis was transferred to our tertiary care center for progressive encephalopathy and concern for nonconvulsive status epilepticus.

Three weeks prior to admission, he was prescribed a 10-day course of levofloxacin for presumed sinusitis. As cough and postnasal drip did not improve, he was treated with two courses of methylprednisolone in addition to levofloxacin and subsequently clarithromycin. Two days prior to presentation, he developed headaches and sinus pressure and complained about "feeling off," epigastric pain, and dry heaving. The night prior to presentation, he awoke frequently throughout the night with progressive confusion. The patient did not have fever, chills, or sweats. His family reported 
a history of anxiety, panic attacks, claustrophobia, and anger outbursts, with the last notable outburst having occurred about 6 months earlier. Of note, coworkers found a collection of various over-the-counter vitamins and nutritional supplements at the patient's desk.

Given increasing confusion and agitation, the patient was taken to another hospital where he became progressively more obtunded, requiring intubation for airway protection. He was empirically started on vancomycin, ceftriaxone, and acyclovir for concern of infectious meningoencephalitis. CSF analysis revealed elevated total protein at $53 \mathrm{mg} / \mathrm{dL}$, glucose of $160 \mathrm{mg} / \mathrm{dL}$ (serum glucose: $240 \mathrm{mg} / \mathrm{dL}$ ), no pleocytosis $\left(1 \mathrm{WBC} / \mathrm{mm}^{3}\right)$, negative CSF VDRL, negative West nile virus IgM and IgG, negative cryptococcal antigen, and negative fungal and bacterial cultures. Due to development of teeth grinding and concern for subclinical seizure activity, a routine electroencephalogram (EEG) was performed that revealed no seizure activity, however findings consistent with global cerebral dysfunction. He was started on phenytoin and subsequently on levetiracetam. An MRI of the brain showed chronic mild periventricular white matter hyperintensities but no acute findings. He was transferred to our tertiary care center on hospital day 3 for concern of nonconvulsive status epilepticus.

On examination, he was intubated and mechanically ventilated, with no abnormal general physical findings. On neurological examination, he was comatose, with intact brainstem reflexes and extensor posturing in all extremities to central stimulation. Initial laboratory work was notable for normal liver function panel, serum ammonia $120 \mu \mathrm{g} / \mathrm{dL}$ (reference range 65-107 $\mu \mathrm{g} / \mathrm{dL}$ ), and negative hepatitis panel, as well as respiratory alkalosis. Repeat CSF analysis showed $3 \mathrm{WBC} / \mathrm{mm}^{3}$ (76\% neutrophils), $1 \mathrm{RBC} / \mathrm{mm}^{3}$, lactic acid $2.6 \mathrm{meq} / \mathrm{L}$, total protein $28.5 \mathrm{mg} / \mathrm{dL}$, and negative herpes simplex virus PCR as well as negative enterovirus panel, and acyclovir was discontinued. Treatment with lactulose was begun, but serum ammonia rapidly rose to $494 \mu \mathrm{g} / \mathrm{dL}$ within a few hours of arrival to our hospital. Rifaximin and L-carnitine were added, and emergent hemodialysis was initiated.

An MRI of the brain on day of transfer showed extensive areas of restricted diffusion with associated FLAIR hyperintensity involving bilateral temporal lobes and bilateral insular, bilateral frontal, and parietal regions in cortical and subcortical areas and diffuse mild effacement of the cerebral sulci (Figure 1), without enhancing lesions. Continuous electroencephalogram (cEEG) for a duration of 96 hours showed continuous, irregular, generalized low voltage slowing (Figure 2); however, intermittently observed facial and lip twitching did not have an electrographic correlate on cEEG. The MRI findings of symmetric grey matter involvement in the abovementioned areas, and in absence of hypoxic-ischemic insult and seizure activity, were deemed most consistent with hyperammonemic encephalopathy.

The patient underwent continuous venous hemofiltration for two consecutive days followed by regular hemodialysis for two days, with sustained correction of ammonia to levels between 29 and $67 \mu \mathrm{g} / \mathrm{dL}$ after day 3. Liver function panel and coagulation parameters remained within normal limits. Due to poor neurological examination and concern for elevated intracranial pressure (ICP), an ICP monitor was placed, revealing three occurrences of ICP elevation to $28-30 \mathrm{mmHg}$, which were treated effectively with mannitol boluses. The patient's nutrition was modified to a low protein, high glucose formula to avoid catabolism of endogenous protein. Two days after normalization of ammonia levels, the patient opened his eyes and started to follow commands and move his extremities, and the ICP monitor was discontinued. He was extubated on hospital day 10, at which time he was alert and conversant, without focal motor deficits, yet remained confused.

A computed tomography of the abdomen with venogram did not reveal a portosystemic shunt. An amino acid panel to evaluate inborn errors of metabolism, specifically ureacycle disorders, was sent (see Table 1). Urine orotic acid excretion was significantly elevated $(>900 \mathrm{mmol} / \mathrm{mol}$ creatinine; reference range: $<2$ ), strongly indicative of a biochemical diagnosis of ornithine transcarbamylase (OTC) deficiency. Genetic testing revealed a pathogenic variant, c.118 C > T, which has been described to cause a mild form of OTC deficiency [3]. In our patient, OTC deficiency was likely unmasked due to a combination of factors: treatment with antibiotics, intake of multiple vitamin supplements, which included red yeast rice, treatment with steroids, and potential interactions of red yeast rice with antibiotics and other herbal supplements.

The patient was maintained on low protein diet and discharged to rehabilitation on hospital day 20 and to home 6 days later, with persistent mild cognitive impairment especially in fluency and memory. A repeat MRI of the brain was obtained 3 weeks after the initial MRI and showed interval decrease in diffusion restriction and FLAIR hyperintensities and resolution of the diffuse mild effacement of the cerebral sulci. He continued to improve clinically and returned to work full time 10 weeks after his initial presentation. At a 3-month follow-up visit, he had returned to his premorbid baseline functional status. At a 6-month follow-up visit, he continued to do well with maintenance of low normal ammonia levels through low protein diet.

\section{Pathophysiology of Hyperammonemia}

3.1. Ammonia in the Healthy Human. Main sources of ammonia are colon (through bacterial metabolism of proteins and urea) and small intestine (through bacterial degradation of glutamine) [4]. In a healthy human, the main metabolic route is uptake of ammonia by periportal hepatocytes followed by urea synthesis via the urea cycle [5]. Ammonia that escapes this pathway is converted to glutamine in perivenous hepatocytes [6]. Hepatic transformation of ammonia into urea and subsequent excretion of urea via colon or kidneys prevent entrance of ammonia into the systemic circulation [2]. If the hepatic metabolic capacity is exceeded, or if ammonia bypasses the liver by shunting of blood, circulating ammonia levels increase and elimination of ammonia is shifted to kidneys, brain, and skeletal muscle. Ammonia that reaches the brain can be metabolized by forming glutamine from glutamate [2], mostly in astrocytes with subsequent transfer 


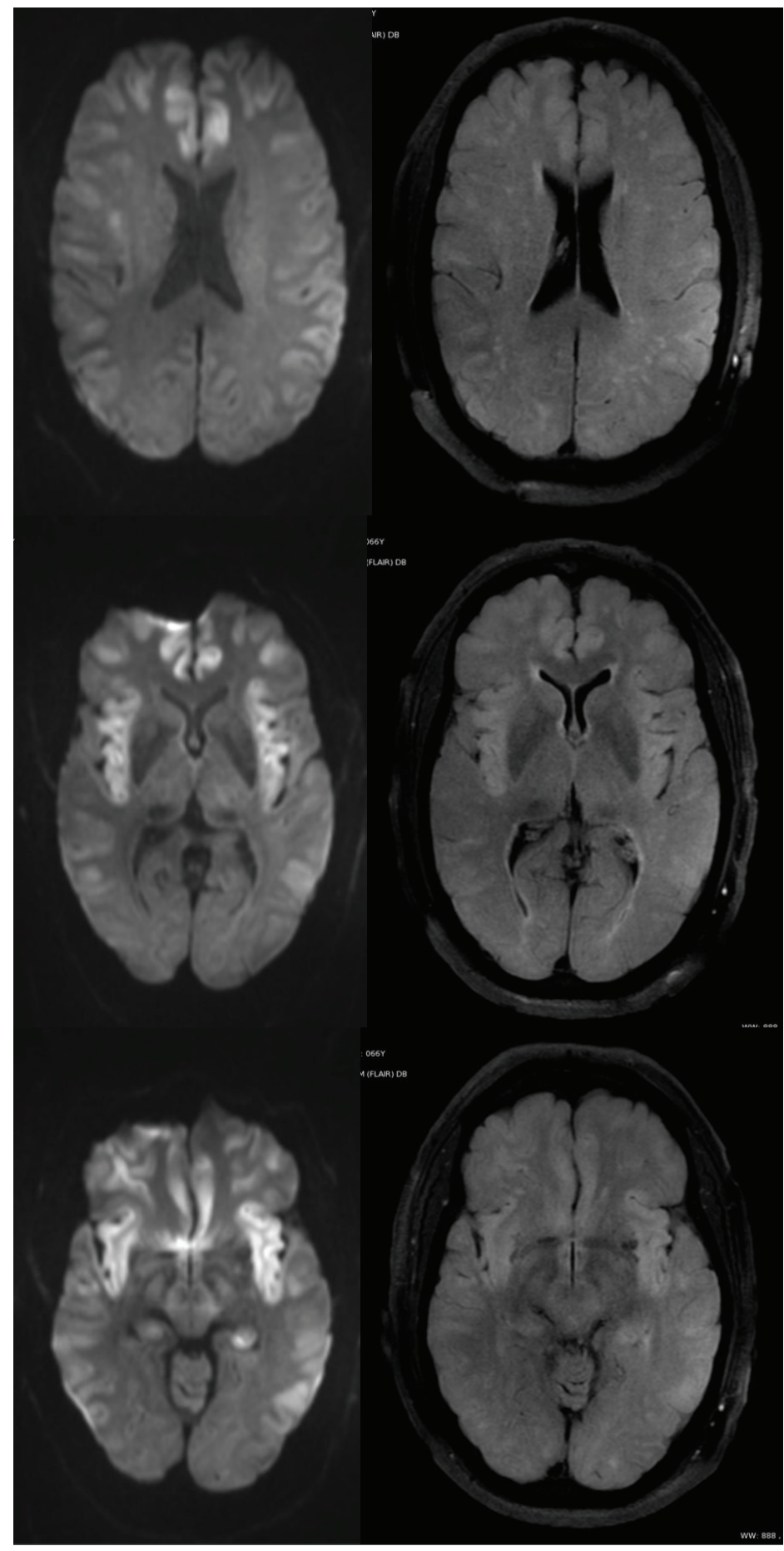

(a)

(b)

FIGURE 1: MRI of the brain ((a) DWI sequence, (b) FLAIR sequence) on day 3 after presentation, showing extensive areas of restricted diffusion with associated FLAIR hyperintensity involving bilateral temporal lobes, bilateral insular, bilateral frontal, and parietal regions in cortical and subcortical areas and diffuse mild effacement of the cerebral sulci.

of glutamine to neurons, and deamination of glutamine resulting in formation of the neurotransmitter glutamate. Muscle adds to the detoxification process by ammonia uptake and synthesis of glutamine [4]. Ammonia excretion through the kidneys, usually accounting for excretion of $30 \%$ of ammonia, can be upregulated to $70 \%$ [2].
3.2. Ammonia in the Brain and Pathophysiology of Hyperammonemic Encephalopathy. Ammonia penetrates the bloodbrain barrier through either passive diffusion or mediated transport [7]. While the exact pathogenesis of neurotoxicity is still elusive, ammonia is believed to play a major role by affecting neuronal function as well as creation of brain edema, each 


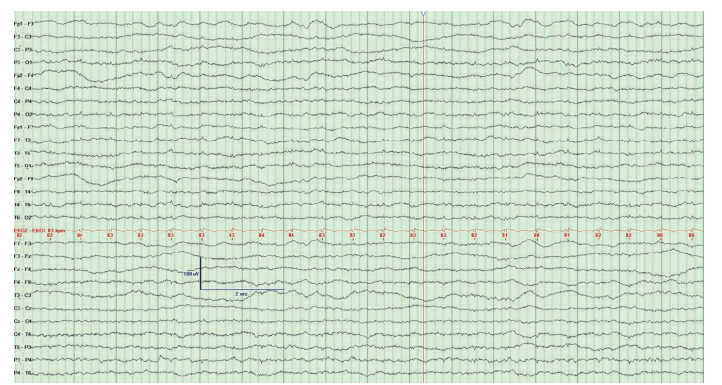

FIGURE 2: EEG on hospital day 3, showing theta frequency slowing and artifacts from spontaneous horizontal and vertical eye movements but no triphasic activity and no epileptiform activity. HFF $70 \mathrm{~Hz}, \mathrm{LFF} 1 \mathrm{~Hz}$; sensitivity and time base indicated on image.

of which can contribute to the development of encephalopathy. Ammonia directly affects neuronal electric activity by inhibiting the generation of both excitatory and inhibitory postsynaptic potentials [8]. Increased cerebral uptake of neutral amino acids, resulting from increased amino acid transport in setting of hyperammonemia, can disturb synthesis of the neurotransmitters dopamine, norepinephrine, and serotonin [9]. Enhanced ammonia metabolism in astrocytes leads to an increase in their production of reactive nitrogen or oxygen species [2] and increased intracellular osmolarity, eventually resulting in brain edema [10]. Furthermore, elevated extracellular glutamate levels, a result of ammoniainduced glutamate release and impaired glutamate clearance, may cause overstimulation of N-methyl-D-aspartate (NMDA) receptors. NMDA receptor activation then triggers nitric oxide synthetase which leads to increased synthesis of the vasodilator nitric oxide [11]. Subsequent resultant intracerebral vasodilatation may contribute to the increase in intracranial pressure. Additionally, astrocyte swelling triggers inflammatory cascades, apoptosis, and metabolic pathways that lead to elevated lactate, cerebral edema, and loss of cerebral autoregulation [12].

3.3. Noncirrhotic Hyperammonemia. The differential diagnosis of hyperammonemia that is not associated with severe liver disease largely falls into one of two categories: increased ammonia production or decreased ammonia elimination.

3.4. Increased Ammonia Production. Increased ammonia production has been observed in hematooncological disorders, organ transplantation, infections, or states of increased catabolism or protein load. The exact mechanism for hyperammonemia in patients with hematooncological disorders is unknown. Myeloma cells have been shown to produce excess ammonia due to increased amino acid metabolism, and plasma cell infiltration of the liver can lead to a portosystemic shunt $[13,14]$. In leukemia patients, occurrence of idiopathic hyperammonemia has been described hours to days after initiation of intensive chemotherapy, often followed by progression to coma and death [15]. Similarly, severe hyperammonemia with frequent lethal course has been described in patients with hematological malignancies treated with
TABLE 1: Results of serum amino acid testing.

\begin{tabular}{|c|c|c|}
\hline Amino acid & $\begin{array}{c}\text { Result } \\
(\mathrm{nmol} / \mathrm{mL})\end{array}$ & $\begin{array}{c}\text { Reference range } \\
(\mathrm{nmol} / \mathrm{mL})\end{array}$ \\
\hline Phosphoserine & 0 & $<18$ \\
\hline Phosphoethanolamine & $<2$ & $<12$ \\
\hline Taurine & $24(L)$ & $42-156$ \\
\hline Asparagine & 40 & $37-92$ \\
\hline Serine & $47(L)$ & $63-187$ \\
\hline Hydroxyproline & $2(L)$ & $4-29$ \\
\hline Glycine & $93(L)$ & $126-490$ \\
\hline Glutamine & 892 & $371-957$ \\
\hline Aspartic acid & 2 & $<7$ \\
\hline Ethanolamine & $<7$ & $<67$ \\
\hline Histidine & 61 & $39-123$ \\
\hline Threonine & $43(L)$ & $85-231$ \\
\hline Citrulline & 22 & $17-46$ \\
\hline Sarcosine & 1 & $<5$ \\
\hline Beta-alanine & 13 & $<29$ \\
\hline Alanine & $88(L)$ & $200-579$ \\
\hline Glutamic acid & 43 & $13-113$ \\
\hline 1-Methylhistidine & 0 & $<28$ \\
\hline 3-Methylhistidine & 3 & $2-9$ \\
\hline Argininosuccinic acid & 0 & $<2$ \\
\hline Carnosine & 0 & $<1$ \\
\hline Anserine & 0 & $<1$ \\
\hline Homocitrulline & 1 & $<2$ \\
\hline Arginine & $28(L)$ & $32-120$ \\
\hline Alpha-aminoadipic acid & 2 & $<3$ \\
\hline Gamma-amino-n-butyric acid & 0 & $<2$ \\
\hline Beta-aminoisobutyric acid & 1 & $<5$ \\
\hline Alpha-amino-n-butyric acid & $77(H)$ & $9-37$ \\
\hline Hydroxylysine & 0 & $<2$ \\
\hline Proline & $81(L)$ & $97-368$ \\
\hline Ornithine & $13(L)$ & $38-130$ \\
\hline Cystathionine & $<1$ & $<5$ \\
\hline Cystine & 36 & 3-95 \\
\hline Lysine & $283(H)$ & $103-255$ \\
\hline Methionine & 41 & $4-44$ \\
\hline Valine & 179 & $136-309$ \\
\hline Tyrosine & 40 & $31-90$ \\
\hline Isoleucine & 54 & $36-107$ \\
\hline Leucine & 93 & $68-183$ \\
\hline Phenylalanine & 48 & $35-80$ \\
\hline Tryptophan & 35 & $29-77$ \\
\hline Allo-isoleucine & 0 & $<5$ \\
\hline
\end{tabular}

bone marrow transplantation [16]. Hyperammonemia has also been described in rare cases after heart-lung or lung transplantation [17, 18]. Pathogenesis of hyperammonemia in these situations is believed to be multifactorial involving 
increased protein catabolism, parenteral nutrition, gastrointestinal hemorrhage, sepsis or mucositis [19], and transient acquired enzyme reductions affecting urea synthesis [20], as well as drug effects from chemotherapy agents [20].

Infections with urea-producing bacteria (Proteus mirabilis, Escherichia coli, Klebsiella species, Providencia rettgeri, Morganella morganii, and diphtheroids) can lead to noncirrhotic hyperammonemic encephalopathy, mostly reported in children with congenital urinary tract abnormalities and urinary stasis [21-23]. However, cases have been reported in adults with urinary retention and neurogenic bladder, or even in absence of urinary tract abnormalities [24]. Ammonia production in the setting of urinary tract infection alkalinizes the urine with subsequent increase of the fraction of ammonium ions [21]. The ammonium ion is less permeable than neutral ammonia and cannot diffuse back into urine and hence escapes detoxification in the liver by uptake into the systemic circulation via venous drainage from the bladder [23]. Furthermore, systemic mycobacterium or mycoplasma infections in organ recipients as well as herpes simplex virus infection in neonates have been described to lead to hyperammonemia [25-27].

Increased ammonia production also occurs during increased muscle catabolism, such as with seizures, starvation, or trauma $[5,28,29]$. However, symptomatic hyperammonemia usually only occurs in patients with underlying urea-cycle disorders [29]. Similarly, total parenteral nutrition (TPN), which contains a high protein load, has been reported to unmask a long-term asymptomatic urea-cycle disorder [30] or occur with TPN containing only essential amino acids with subsequently impaired ammonia detoxification due to absence of ornithine [31].

3.5. Decreased Ammonia Elimination. Inborn errors of metabolism (IEMs) that can cause hyperammonemia include urea-cycle disorders, organic acidurias, carnitine deficiency from defects in fatty acid oxidation, dibasic aminoaciduria, and defects in pyruvate metabolism [6]. While most IEMs present during the neonatal period or early childhood, some, especially urea-cycle disorders, can present in adults. Every enzyme in the urea cycle, carbamoyl phosphate synthetase (CPS), ornithine transcarbamylase, argininosuccinate synthetase (ASS), argininosuccinic acid lyase, and arginase, can be affected by an inherited deficiency [32-34]. OTC deficiency, which is inherited in an X-linked recessive manner, is the most common one, with an estimated prevalence of $1: 14.000$ [35]. Late presentations and phenotypic variance are widely known for OTC deficiency. Mild OTC deficiency can remain largely asymptomatic until an inciting event unmasks the deficiency and leads to symptomatic hyperammonemia $[36,37]$. CPS deficiency, $\mathrm{N}$-acetyl glutamine synthetase, and type II citrullinemia also can be present in adulthood [3840]. Precipitating factors for clinical manifestation of these deficiencies include infections [23, 39], TPN [30], gastrointestinal hemorrhage [41], or valproate intake [42].

Ammonia elimination can be significantly decreased in presence of a portosystemic shunt. Congenital portosystemic shunts are a rare cause of noncirrhotic hyperammonemia, and shunt volume determines time of manifestation, with increased prevalence in patients above the age of 60 [43]. An acquired form of noncirrhotic portosystemic shunt that can lead to hyperammonemic encephalopathy is portal vein thrombosis [44].

Ureterosigmoidostomy is another anatomic situation that can lead to hyperammonemia, due to increased ammonia formation by bacterial degradation after urine excretion directly into the sigmoid colon [45]. While this can occur in liver-healthy individuals, for example, due to coprostasis or infection with urea-splitting bacteria [46], most cases occur in the setting of hepatic failure [47].

3.6. Drug-Induced Hyperammonemia. Drug-induced hyperammonemia can result from interference with the urea cycle or enhancement of renal release of ammonia into the systemic circulation. Valproic acid is the most well known [48], but others include carbamazepine [49], sulfadiazine [50], ribavirin [51], salicylates [52], and glycine [53].

The exact pathogenesis of hyperammonemia due to valproic acid is unclear, but it has been suggested that the mechanism is through inhibition of glutamate uptake by astrocytes [48]. The reported prevalence of valproic acid induced hyperammonemia is as high as $35-45 \%$ and seems to be higher in patients with carnitine deficiency or with congenital urea-cycle enzymatic defects [54]. Symptoms can present as early as 2 weeks into therapy [6] or as late as several years later [55]. Patients can be asymptomatic with mildly elevated liver enzymes or present with cognitive dysfunction, coma, or severe hepatotoxicity $[48,56]$. Serum valproic acid levels can be normal and do not correlate with the level of hyperammonemia or symptoms [56]. Furthermore, ammonia levels do not correlate with the severity of encephalopathy [32].

\section{Clinical Presentation of Hyperammonemia}

Symptoms can range from mild, such as irritability, headache, and vomiting, to severe with encephalopathy, seizures, ataxia, and coma. Depending on the underlying etiology, the symptoms can fluctuate and episodically occur, often precipitated by increased protein intake, drugs, or infections [57]. Psychiatric manifestations, such as manic episodes or psychosis, may be seen in chronic manifestations of lateonset presentations of inborn errors of metabolism [55, 57]. Seizures, cerebral edema, and herniation are manifestations of acute hyperammonemia and usually occur with ammonia levels exceeding $200 \mu \mathrm{mol} / \mathrm{L}$ [58]. The difference between acute and chronic hyperammonemia is believed to lie in the effect of glutamine on the brain [5]. In patients with acute liver failure, a strong association of arterial ammonia levels higher than $200 \mu \mathrm{g} / \mathrm{dL}$ with cerebral herniation has been shown [59].

\section{Diagnosis}

5.1. Laboratory Workup. To accurately determine the serum ammonia level, the blood sample must be obtained and handled correctly. Hemolysis, inappropriate handling and 
transportation of the specimen (the specimen needs to be placed on ice), and delayed analysis all can falsely elevate ammonia levels. Both venous and arterial blood can be sampled for measurement of ammonia [60]. In patients with hepatic failure who are suspected to have hepatic encephalopathy, measurement of ammonia remains controversial. While there is a correlation between ammonia levels and severity of hepatic encephalopathy, absolute elevation of ammonia is inconsistent [60]. Furthermore, hepatic encephalopathy is only directly related to arterial ammonia levels up to about twofold increase above normal; beyond that, the grade of hepatic encephalopathy is dependent on the partial pressure of gaseous ammonia rather than total serum ammonia [61]. However, this may not necessarily be applicable to hyperammonemia in absence of hepatic failure.

A value of more than $100 \mu \mathrm{mol} / \mathrm{L}(175 \mu \mathrm{g} / \mathrm{dL})$ in older children and adults should trigger further investigation, especially in setting of normal liver function [62]. For isolated hyperammonemia, first evaluations include acid-base status, serum bicarbonate, sodium, chloride, and urine ketones [63]. Acidosis raises the possibility of organic acidemias whereas respiratory alkalosis may be an indication of a ureacycle disorder. In absence of acidosis, the concentrations of amino acids in blood and urine should be evaluated [62]. A nondiagnostic amino acid profile would trigger evaluation for urine orotic acid, as orotic aciduria is found in patients with OTC deficiency. If both amino acid profile and orotic acid testing are nonrevealing, the diagnosis likely is CPS deficiency or, much more rarely, NAGS deficiency [62]. In patients with episodic symptoms, the diagnosis may be elusive until reevaluation during crisis [63]. Ultrasonography, abdominal computed tomography, and magnetic resonance imaging can all demonstrate portohepatic shunts [64]. A liver biopsy should be considered to measure enzyme levels in hepatocytes if an inborn error of metabolism is suspected [32]. Liver biopsy can furthermore be invaluable in excluding cirrhosis of any cause or diagnose other hepatic diseases.

5.2. Neuroimaging. Magnetic resonance imaging (MRI) has provided insight into characteristic patterns observed in hyperammonemic encephalopathy. Diffusion restriction is found predominantly cortical and often diffuse and symmetric, with corresponding areas of FLAIR hyperintensities [65]. Insular cortex and cingulate gyrus are most commonly affected, but it remains unclear why these areas are particularly susceptible to hyperammonemia $[66,67]$. Thalamus, parietal, frontal, temporal, or occipital cortices involvement can be seen in variable extent and may be asymmetric $[66,67]$. There is one case report of thalami and basal ganglia involvement, and involvement of the occipital cortices is considered rare [65]. In neonatal cases of inborn errors of metabolism with resultant hyperammonemia, a slightly different pattern was described with involvement of the lentiform nuclei, insular sulci, and perirolandic regions possibly due to those areas being more metabolically active in the neonatal period [68]. Radiographic abnormalities may be detected even if ammonia is only slightly elevated [65, 66]. The extent of the imaging findings may depend on severity and duration of hyperammonemia and predisposing susceptibility to the metabolic insult [68]. Cortical changes in hyperammonemic encephalopathy have been shown to be potentially reversible [69] but can also result in variable amounts of atrophy in the cingulate and insular cortex [66, 68].

Differential diagnosis includes posterior reversible encephalopathy syndrome, seizure activity, metabolic and hepatic encephalopathy, and diffuse hypoxic-ischemic injury [66]. Exclusion of an overlapping effect of hypoxic injury or seizure activity on imaging findings may be difficult, but very symmetric involvement of cingulate and insular gyri favor a toxic encephalopathy [66].

\section{Treatment}

Acute treatments of hyperammonemia are geared towards lowering the blood ammonia level and manage seizures, cerebral edema, and elevated intracranial pressure. Continuing treatments may target the specific etiology of hyperammonemia such as inborn errors of metabolism and vary with the condition.

6.1. Ammonia-Lowering Treatment. Nonabsorbable disaccharides, which decrease intestinal ammonia production and absorption, are an established first-line therapy for hepatic encephalopathy and the mainstay of treatment for chronic encephalopathy; however, they do not affect mortality [70]. Antibiotics that decrease enteric ammonia production by reducing the amount of urease-producing bacteria are another treatment option. Neomycin, an antibiotic and glutaminase inhibitor, has been FDA approved for use in acute hepatic encephalopathy but is also commonly used with lactulose as off-label treatment for chronic encephalopathy [71]. Rifaximin, a nonabsorbable antibiotic derivative, is used as first-line or in addition to nonabsorbable disaccharides in acute or chronic encephalopathy [72]. Sodium benzoate or sodium phenyl acetate enhances alternative pathways of ammonia metabolism and subsequent excretion via urine [33]. Emergent hemodialysis can be used to rapidly, though temporarily, decrease serum ammonia, with conventional hemodialysis offering the highest ammonium clearance rate of all dialysis methods [73].

6.2. General Management. Data and practices of management for elevated ICP and cerebral edema are largely available from observations in (fulminant) hepatic failure and may not apply to isolated hyperammonemia. Usual management includes hyperosmolar agents and propofol sedation [74]. While mannitol used to be the mainstay of therapy despite doubts about efficacy [75], hypertonic saline has gained more attention [76]. For medically refractory intracranial hypertension, decompressive craniectomy can be considered for a potential positive outcome [77]. ICP monitoring and transcranial Doppler sonography may be used to assist in diagnosis and monitoring of treatment effect [78], but ICP monitoring has been shown to have potential detrimental effects in absence of mortality benefit [79]. 
6.3. Specific and Ongoing Treatments. Introduction of a diet with a favorable calorie-to-nitrogen ratio and restriction of exogenous protein is a supporting measure [80]. L-Carnitine plays a critical role in the intermediary metabolism of fatty acids and their transport across mitochondrial membranes and has been shown to be of use in treatment of inborn errors of metabolism [81]. Supplementation with L-carnitine in urea-cycle disorders may lower the frequency of attacks [82]. While primary treatment for valproic acid induced hyperammonemia is withdrawal of the drug and limitation of potentiating drugs such as phenobarbital and phenytoin [83], treatment of valproic acid induced hyperammonemia with Lcarnitine has been reported to improve both symptoms and survival [84].

L-Ornithine-L-aspartate increases muscle ammonia metabolism and has been shown to be beneficial in clinically manifest hepatic encephalopathy [85]. Arginine, the immediate precursor of ornithine, can serve as treatment of hyperammonemia in urea-cycle disorders by replenishing the urea-cycle substrates [86]. Liver transplantation is an important treatment modality in urea-cycle disorders, with high survival rates that are superior to survival in liver transplantation for other diseases [87]. Portosystemic shunts may be obliterated surgically or by interventional radiological techniques but, depending on the type, may also require liver transplantation [88].

\section{Discussion}

Several case reports have described acute onset of hyperammonemia due to late-onset inborn errors of metabolism in previously healthy adolescents or adults. The majority of these cases are fatal [89-92]. Panlaqui et al. report a case of a 48-year-old man with preceding subacute cognitive decline and sudden encephalopathy, an ammonia level of $390 \mu \mathrm{mol} / \mathrm{L}$, MRI findings of diffuse cortical edema and T2 hyperintensities, successfully treated with hemodialysis, and however persistent significant cognitive deficits at 6-month follow-up [93]. Mahmood and Nugent describe a 35-year-old woman with late-onset OTC deficiency unmasked by gastrointestinal hemorrhage, presenting with encephalopathy [94]. A CT of the head in this patient was reportedly normal, and ammonia level was $593 \mu \mathrm{g} / \mathrm{dL}$ [94]. Wendell et al. report successful management of ICP in a 37-year-old female with known OTC deficiency, ammonia level of $904 \mu \mathrm{mol} / \mathrm{L}$, and progressive cerebral edema on head CT eventually requiring decompressive craniectomy [77], with good outcome. UKing-Im et al. discuss a series of four patients with acute adult hyperammonemic encephalopathy who presented with seizures and decreased level of consciousness and plasma ammonia levels between 55 and $168 \mu \mathrm{mol} / \mathrm{L}$ [66]. Unlike our patient, these four described patients all had severe systemic disease or hepatic failure, namely, (1) status after heartlung transplantation with multiple organ dysfunction and sepsis, (2) fulminant acute hepatic failure, (3) severe sepsis in setting of chronic cirrhosis, and (4) severe sepsis in a hepatic transplant patient with hepatorenal syndrome. All four patients had MRI findings delineating the characteristic findings of hyperammonemia. Only one patient had a good outcome [66]. Rosario et al. describe three patients with cirrhosis, alcoholic hepatitis, and acetaminophen hepatotoxicity [67], with characteristic MRI findings. Only the patient with acetaminophen toxicity, whose ammonia level had been $217 \mu \mathrm{mol} / \mathrm{L}$, had a good outcome [67].

We therefore present a novel and unique case of an adult with late-onset OTC deficiency, with very severe clinical manifestation and very high ammonia levels, as well as extensive MRI findings characteristic for hyperammonemic encephalopathy. Absence of status epilepticus and only mild transient ICP elevation point to hyperammonemia as the main factor in causing this patient's encephalopathy and imaging findings. Our case highlights that extensive imaging findings can be reversible in late-onset OTC deficiency and that outcome can be excellent if correctly diagnosed and aggressively and rapidly treated.

\section{Conclusion}

Hyperammonemia should be considered in the differential diagnosis for encephalopathy and seizures, especially in presence of MRI findings of bilateral symmetric involvement of insular and cingulate cortices. In absence of hepatic dysfunction, hyperammonemia can be caused by increased ammonia production or decreased ammonia excretion. Aggressive management with ammonia-lowering measures, cerebral edema, and increased intracranial pressure is warranted.

\section{Ethical Approval}

Permission to publish this case report was granted by the local Institutional Review Board.

\section{Competing Interests}

The authors declare no competing interests.

\section{References}

[1] P. Ferenci, "Brain dysfunction in fulminant hepatic failure," Journal of Hepatology, vol. 21, no. 4, pp. 487-490, 1994.

[2] H. Cichoz-Lach and A. Michalak, "Current pathogenetic aspects of hepatic encephalopathy and noncirrhotic hyperammonemic encephalopathy," World Journal of Gastroenterology, vol. 19, no. 1, pp. 26-34, 2013.

[3] L. Caldovic, I. Abdikarim, S. Narain, M. Tuchman, and H. Morizono, "Genotype-phenotype correlations in ornithine transcarbamylase deficiency: a mutation update," Journal of Genetics and Genomics, vol. 42, no. 5, pp. 181-194, 2015.

[4] S. W. M. Olde Damink, R. Jalan, and C. H. C. Dejong, "Interorgan ammonia trafficking in liver disease," Metabolic Brain Disease, vol. 24, no. 1, pp. 169-181, 2009.

[5] A. S. Clay and B. E. Hainline, "Hyperammonemia in the ICU," Chest, vol. 132, no. 4, pp. 1368-1378, 2007.

[6] W. R. Treem, "Inherited and acquired syndromes of hyperammonemia and encephalopathy in children," Seminars in Liver Disease, vol. 14, no. 3, pp. 236-258, 1994. 
[7] P. Ott and H. Vilstrup, "Cerebral effects of ammonia in liver disease: current hypotheses," Metabolic Brain Disease, vol. 29, no. 4, pp. 901-911, 2014.

[8] W. Raabe, "Ammonium ions abolish excitatory synaptic transmission between cerebellar neurons in primary dissociated tissue culture," Journal of Neurophysiology, vol. 68, no. 1, pp. 9399, 1992.

[9] J. E. Fischer, N. Yoshimura, A. Aguirre et al., "Plasma amino acids in patients with hepatic encephalopathy. Effects of amino acid infusions," The American Journal of Surgery, vol. 127, no. 1, pp. 40-47, 1974.

[10] A. T. Blei, S. Olafsson, G. Therrien, and R. F. Butterworth, "Ammonia-induced brain edema and intracranial hypertension in rats after portacaval anastomosis," Hepatology, vol. 19, no. 6, pp. 1437-1444, 1994.

[11] A. T. Blei and F. S. Larsen, "Pathophysiology of cerebral edema in fulminant hepatic failure," Journal of Hepatology, vol. 31, no. 4, pp. 771-776, 1999.

[12] L. Hertz and G. Kala, "Energy metabolism in brain cells: effects of elevated ammonia concentrations," Metabolic Brain Disease, vol. 22, no. 3-4, pp. 199-218, 2007.

[13] L. Kwan, C. Wang, and L. Levitt, "Hyperammonemic encephalopathy in multiple myeloma," The New England Journal of Medicine, vol. 346, no. 21, pp. 1674-1675, 2002.

[14] T. Otsuki, O. Yamada, H. Sakaguchi et al., "In vitro excess ammonia production in human myeloma cell lines," Leukemia, vol. 12, no. 7, pp. 1149-1158, 1998.

[15] R. B. Mitchell, J. E. Wagner, J. E. Karp et al., "Syndrome of idiopathic hyperammonemia after high-dose chemotherapy: review of nine cases," The American Journal of Medicine, vol. 85, no. 5, pp. 662-667, 1988.

[16] S. M. Davies, E. Szabo, J. E. Wagner, N. K. C. Ramsay, and D. J. Weisdorf, "Idiopathic hyperammonemia: a frequently lethal complication of bone marrow transplantation," Bone Marrow Transplantation, vol. 17, no. 6, pp. 1119-1125, 1996.

[17] G. R. Lichtenstein, Y.-X. Yang, F. A. Nunes et al., "Fatal hyperammonemia after orthotopic lung transplantation," Annals of Internal Medicine, vol. 132, no. 4, pp. 283-287, 2000.

[18] E. M. Yoshida, D. N. Ostrow, S. R. Erb, and G. Fradet, "Hyperammonemia after heart-lung transplantation," Gastroenterology, vol. 112, no. 6, p. 2162, 1997.

[19] M. del Rosario, S. L. Werlin, and S. J. Lauer, "Hyperammonemic encephalopathy after chemotherapy: survival after treatment with sodium benzoate and sodium phenylacetate," Journal of Clinical Gastroenterology, vol. 25, no. 4, pp. 682-684, 1997.

[20] K. H. Metzeler, S. Boeck, B. Christ et al., "Idiopathic hyperammonemia (IHA) after dose-dense induction chemotherapy for acute myeloid leukemia: case report and review of the literature," Leukemia Research, vol. 33, no. 7, pp. e69-e72, 2009.

[21] H.-K. Cheang, L. Rangecroft, N. D. Plant, and A. A. M. Morris, "Hyperammonaemia due to Klebsiella infection in a neuropathic bladder," Pediatric Nephrology, vol. 12, no. 8, pp. 658-659, 1998.

[22] D. A. Diamond, A. Blight, and P. G. Ransley, "Hyperammonemic encephalopathy: a complication associated with the prune belly syndrome," The Journal of Urology, vol. 142, no. 2, part 1, pp. 361-362, 1989.

[23] B. Samtoy and M. M. DeBeukelaer, "Ammonia encephalopathy secondary to urinary tract infection with Proteus mirabilis," Pediatrics, vol. 65, no. 2, pp. 294-297, 1980.
[24] B. De Jonghe, V. Janier, N. Abderrahim, D. Hillion, J.-C. Lacherade, and H. Outin, "Urinary tract infection and coma," The Lancet, vol. 360, no. 9338, p. 996, 2002.

[25] P. M. Barnes, D. B. Wheldon, C. Eggerding, W. C. Marshall, and J. V. Leonard, "Hyperammonaemia and disseminated herpes simplex infection in the neonatal period," The Lancet, vol. 319, no. 8285, pp. 1362-1363, 1982.

[26] S. Nurmohamed, A. Weenink, H. Moeniralam, C. Visser, and F. Bemelman, "Hyperammonemia in generalized Mycobacterium genavense infection after renal transplantation," American Journal of Transplantation, vol. 7, no. 3, pp. 722-723, 2007.

[27] M. E. Wylam, C. C. Kennedy, N. M. Hernandez et al., "Fatal hyperammonaemia caused by Mycoplasma hominis," The Lancet, vol. 382, no. 9908, p. 1956, 2013.

[28] N. D. Hawkes, G. A. O. Thomas, A. Jurewicz et al., "Non-hepatic hyperammonaemia: an important, potentially reversible cause of encephalopathy," Postgraduate Medical Journal, vol. 77, no. 913, pp. 717-722, 2001.

[29] M. L. Summar, F. Barr, S. Dawling et al., "Unmasked adult-onset urea cycle disorders in the critical care setting," Critical Care Clinics, vol. 21, no. 4, pp. S1-S8, 2005.

[30] D. M. Felig, S. W. Brusilow, and J. L. Boyer, "Hyperammonemic coma due to parenteral nutrition in a woman with heterozygous ornithine transcarbamylase deficiency," Gastroenterology, vol. 109, no. 1, pp. 282-284, 1995.

[31] R. E. Grazer, J. M. Sutton, S. Friedstrom, and F. D. McBarron, "Hyperammonemic encephalopathy due to essential amino acid hyperalimentation," Archives of Internal Medicine, vol. 144, no. 11, pp. 2278-2279, 1984.

[32] I. Laish and Z. Ben Ari, "Noncirrhotic hyperammonaemic encephalopathy," Liver International, vol. 31, no. 9, pp. 12591270, 2011.

[33] S. W. Brusilow and N. E. Maestri, "Urea cycle disorders: diagnosis, pathophysiology, and therapy," Advances in Pediatrics, vol. 43, pp. 127-170, 1996.

[34] B. K. Burton, "Urea cycle disorders," Clinics in Liver Disease, vol. 4, no. 4, pp. 815-830, 2000.

[35] J. A. Arranz, E. Riudor, C. Marco-Marín, and V. Rubio, "Estimation of the total number of disease-causing mutations in ornithine transcarbamylase (OTC) deficiency. Value of the OTC structure in predicting a mutation pathogenic potential," Journal of Inherited Metabolic Disease, vol. 30, no. 2, pp. 217-226, 2007.

[36] P. H. Arn, E. R. Hauser, G. H. Thomas, G. Herman, D. Hess, and S. W. Brusilow, "Hyperammonemia in women with a mutation at the ornithine carbamoyltransferase locus-a cause of postpartum coma," The New England Journal of Medicine, vol. 322, no. 23, pp. 1652-1655, 1990.

[37] Z. Ben-Ari, A. Dalal, A. Morry et al., "Adult-onset ornithine transcarbamylase (OTC) deficiency unmasked by the Atkins' diet," Journal of Hepatology, vol. 52, no. 2, pp. 292-295, 2010.

[38] O. Elpeleg, A. Shaag, E. Ben-Shalom, T. Schmid, and C. Bachmann, "N-acetylglutamate synthase deficiency and the treatment of hyperammonemic encephalopathy," Annals of Neurology, vol. 52, no. 6, pp. 845-849, 2002.

[39] W. D. Lo, H. R. Sloan, J. F. Sotos, and R. J. Klinger, "Late clinical presentation of partial carbamyl phosphate synthetase I deficiency," American Journal of Diseases of Children, vol. 147, no. 3, pp. 267-269, 1993.

[40] F. Ishikawa, M. Nakamuta, M. Kato et al., "Reversibility of serum $\mathrm{NH}_{3}$ level in a case of sudden onset and rapidly progressive case 
of type 2 citrullinemia," Internal Medicine, vol. 39, no. 11, pp. 925-929, 2000.

[41] M. Trivedi, S. Zafar, M. J. Spalding, and S. Jonnalagadda, "Ornithine transcarbamylase deficiency unmasked because of gastrointestinal bleeding," Journal of Clinical Gastroenterology, vol. 32, no. 4, pp. 340-343, 2001.

[42] D. Honeycutt, K. Callahan, L. Rutledge, and B. K. Evans, "Heterozygote ornithine transcarbamylase deficiency presenting as symptomatic hyperammonemia during initiation of valproate therapy," Neurology, vol. 42, no. 3, part 1, pp. 666-668, 1992.

[43] T. Uchino, I. Matsuda, and F. Endo, "The long-term prognosis of congenital portosystemic venous shunt," The Journal of Pediatrics, vol. 135, no. 2, part 1, pp. 254-256, 1999.

[44] S. K. Yadav, A. Srivastava, A. Srivastava et al., "Encephalopathy assessment in children with extra-hepatic portal vein obstruction with MR, psychometry and critical flicker frequency," Journal of Hepatology, vol. 52, no. 3, pp. 348-354, 2010.

[45] A. R. Mundy, "Metabolic complications of urinary diversion," The Lancet, vol. 353, no. 9167, pp. 1813-1814, 1999.

[46] W. S. McDougal, "Metabolic complications of urinary intestinal diversion," The Journal of Urology, vol. 147, no. 5, pp. 1199-1208, 1992.

[47] E. Mortensen, G. Lyng, E. Juhl, J. Egense, and M. Schwartz, "Ammonia-induced coma after ureterosigmoidostomy," The Lancet, vol. 299, no. 7758, p. 1024, 1972.

[48] A. Verrotti, D. Trotta, G. Morgese, and F. Chiarelli, "Valproateinduced hyperammonemic encephalopathy," Metabolic Brain Disease, vol. 17, no. 4, pp. 367-373, 2002.

[49] G. Ambrosetto, R. Riva, and A. Baruzzi, "Hyperammonemia in asterixis induced by carbamazepine: two case reports," Acta Neurologica Scandinavica, vol. 69, no. 3, pp. 186-189, 1984.

[50] G. Sekas and H. S. Paul, "Hyperammonemia and carnitine deficiency in a patient receiving sulfadiazine and pyrimethamine," The American Journal of Medicine, vol. 95, no. 1, pp. 112-113, 1993.

[51] P. Bertrand, A. Faro, P. Cantwell, and A. Tzakis, "Intravenous ribavirin and hyperammonemia in an immunocompromised patient infected with adenovirus," Pharmacotherapy, vol. 20, no. 10, pp. 1216-1220, 2000.

[52] A. L. Makela, H. Lang, and P. Korpela, "Toxic encephalopathy with hyperammonaemia during high-dose salicylate therapy," Acta Neurologica Scandinavica, vol. 61, no. 3, pp. 146-156, 1980.

[53] K. W. Ryder, J. F. Olson, R. J. Kahnoski, R. C. Karn, and T. O. Oei, "Hyperammonemia after transurethral resection of the prostate: a report of 2 cases," The Journal of Urology, vol. 132, no. 5, pp. 995-997, 1984.

[54] J. V. Murphy and K. Marquardt, "Asymptomatic hyperammonemia in patients receiving valproic acid," Archives of Neurology, vol. 39, no. 9, pp. 591-592, 1982.

[55] M.-J. C. C. Dealberto, "Valproate-induced hyperammonaemic encephalopathy: review of 14 cases in the psychiatric setting," International Clinical Psychopharmacology, vol. 22, no. 6, pp. 330-337, 2007.

[56] T. Gerstner, D. Buesing, E. Longin et al., "Valproic acid induced encephalopathy-19 new cases in Germany from 1994 to 2003a side effect associated to VPA-therapy not only in young children," Seizure, vol. 15, no. 6, pp. 443-448, 2006.

[57] G. N. Breningstall, "Neurologic syndromes in hyperammonemic disorders," Pediatric Neurology, vol. 2, no. 5, pp. 253-262, 1986.
[58] J. Vaquero, C. Chung, M. E. Cahill, and A. T. Blei, "Pathogenesis of hepatic encephalopathy in acute liver failure," Seminars in Liver Disease, vol. 23, no. 3, pp. 259-269, 2003.

[59] J. O. Clemmesen, F. S. Larsen, J. Kondrup, B. A. Hansen, and P. Ott, "Cerebral herniation in patients with acute liver failure is correlated with arterial ammonia concentration," Hepatology, vol. 29, no. 3, pp. 648-653, 1999.

[60] J. P. Ong, A. Aggarwal, D. Krieger et al., "Correlation between ammonia levels and the severity of hepatic encephalopathy," American Journal of Medicine, vol. 114, no. 3, pp. 188-193, 2003.

[61] L. Kramer, B. Tribl, A. Gendo et al., "Partial pressure of ammonia versus ammonia in hepatic encephalopathy," Hepatology, vol. 31, no. 1, pp. 30-34, 2000.

[62] G. F. Hoffman, W. L. Nyhan, J. Zschocke, S. G. Kahler, and E. Mayatepek, Inherited Metabolic Disease, Lippincott Williams \& Wilkins, Philadelphia, Pa, USA, 2002.

[63] A. N. Rao, P. Varma, Sumitra, and S. Dhanya, "Hyperammonemia: diagnostic experience at the metabolism laboratory," The Internet Journal of Laboratory Medicine, vol. 1, no. 2, 2005.

[64] A. Watanabe, "Portal-systemic encephalopathy in non-cirrhotic patients: classification of clinical types, diagnosis and treatment," Journal of Gastroenterology and Hepatology, vol. 15, no. 9, pp. 969-979, 2000.

[65] A. de Havenon, K. French, and S. Ansari, "Extensive cortical diffusion restriction in a 50-year-old female with hyperammonemic encephalopathy and status epilepticus," Case Reports in Neurological Medicine, vol. 2014, Article ID 257094, 4 pages, 2014.

[66] J. M. U-King-Im, E. Yu, E. Bartlett, R. Soobrah, and W. Kucharczyk, "Acute hyperammonemic encephalopathy in adults: imaging findings," American Journal of Neuroradiology, vol. 32, no. 2, pp. 413-418, 2011.

[67] M. Rosario, K. McMahon, and P. F. Finelli, "Diffusion-weighted imaging in acute hyperammonemic encephalopathy," The Neurohospitalist, vol. 3, no. 3, pp. 125-130, 2013.

[68] J.-I. Takanashi, A. J. Barkovich, S. F. Cheng et al., "Brain MR imaging in neonatal hyperammonemic encephalopathy resulting from proximal urea cycle disorders," American Journal of Neuroradiology, vol. 24, no. 6, pp. 1184-1187, 2003.

[69] A. Kawata, M. Suda, and H. Tanabe, "Adult-onset type II citrullinemia: clinical pictures before and after liver transplantation," Internal Medicine, vol. 36, no. 6, pp. 408-412, 1997.

[70] B. Als-Nielsen, L. L. Gluud, and C. Gluud, "Nonabsorbable disaccharides for hepatic encephalopathy," Cochrane Database of Systematic Reviews, no. 2, Article ID CD003044, 2004.

[71] M. I. Nevah and M. B. Fallon, "Hepatic encephalopathy, hepatorenal syndrome, hepatopulmonary syndrome and systemic complications of liver disease," in Sleisenger and Fordtran's Gastrointestinal and Liver Disease: Pathophysiology/Diagnosis/Management, M. Feldman, L. S. Friedman, and L. J. Brandt, Eds., pp. 1543-1555, Saunders, Elsevier, Philadelphia, $\mathrm{Pa}, \mathrm{USA}$, 9th edition, 2010.

[72] N. M. Bass, K. D. Mullen, A. Sanyal et al., "Rifaximin treatment in hepatic encephalopathy," The New England Journal of Medicine, vol. 362, no. 12, pp. 1071-1081, 2010.

[73] S. M. Donn, R. D. Swartz, and J. G. Thoene, "Comparison of exchange transfusion, peritoneal dialysis, and hemodialysis for the treatment of hyperammonemia in an anuric newborn infant," The Journal of Pediatrics, vol. 95, no. 1, pp. 67-70, 1979.

[74] V. Mohsenin, "Assessment and management of cerebral edema and intracranial hypertension in acute liver failure," Journal of Critical Care, vol. 28, no. 5, pp. 783-791, 2013. 
[75] V. A. Saraswat, S. Saksena, K. Nath et al., "Evaluation of mannitol effect in patients with acute hepatic failure and acuteon-chronic liver failure using conventional MRI, diffusion tensor imaging and in-vivo proton MR spectroscopy," World Journal of Gastroenterology, vol. 14, no. 26, pp. 4168-4178, 2008.

[76] E. M. Liotta, B. D. Lizza, A. L. Romanova et al., " $23.4 \%$ Saline decreases brain tissue volume in severe hepatic encephalopathy as assessed by a quantitative CT marker," Critical Care Medicine, vol. 44, no. 1, pp. 171-179, 2016.

[77] L. C. Wendell, A. Khan, J. Raser et al., "Successful management of refractory intracranial hypertension from acute hyperammonemic encephalopathy in a woman with ornithine transcarbamylase deficiency," Neurocritical Care, vol. 13, no. 1, pp. 113-117, 2010.

[78] S. Kodali and B. M. McGuire, "Diagnosis and management of hepatic encephalopathy in fulminant hepatic failure," Clinics in Liver Disease, vol. 19, no. 3, pp. 565-576, 2015.

[79] C. J. Karvellas, O. K. Fix, H. Battenhouse, V. Durkalski, C. Sanders, and W. M. Lee, "Outcomes and complications of intracranial pressure monitoring in acute liver failure: a retrospective cohort study," Critical Care Medicine, vol. 42, no. 5, pp. 1157-1167, 2014.

[80] R. M. Cohn and K. S. Roth, "Hyperammonemia, bane of the brain," Clinical Pediatrics, vol. 43, no. 8, pp. 683-689, 2004.

[81] M. Malaguarnera, G. Pistone, M. Astuto et al., "L-Carnitine in the treatment of mild or moderate hepatic encephalopathy," Digestive Diseases, vol. 21, no. 3, pp. 271-275, 2003.

[82] Y. Ohtani, K. Ohyanagi, S. Yamamoto, and I. Matsuda, "Secondary carnitine deficiency in hyperammonemic attacks of ornithine transcarbamylase deficiency," The Journal of Pediatrics, vol. 112, no. 3, pp. 409-414, 1988.

[83] M. F. Camiña, I. Rozas, M. Castro-Gago, J. M. Paz, C. Alonso, and S. Rodriguez-Segade, "Alteration of renal carnitine metabolism by anticonvulsant treatment," Neurology, vol. 41, no. 9, pp. 1444-1448, 1991.

[84] T. P. Bohan, E. Helton, I. McDonald et al., "Effect of L-carnitine treatment for valproate-induced hepatotoxicity," Neurology, vol. 56, no. 10, pp. 1405-1409, 2001

[85] Q. Jiang, X.-H. Jiang, M.-H. Zheng, and Y.-P. Chen, "LOrnithine-L-aspartate in the management of hepatic encephalopathy: a meta-analysis," Journal of Gastroenterology and Hepatology, vol. 24, no. 1, pp. 9-14, 2009.

[86] S. W. Brusilow, "Arginine, an indispensable amino acid for patients with inborn errors of urea synthesis," Journal of Clinical Investigation, vol. 74, no. 6, pp. 2144-2148, 1984.

[87] D. Morioka, M. Kasahara, Y. Takada et al., "Current role of liver transplantation for the treatment of urea cycle disorders: a review of the worldwide English literature and 13 cases at Kyoto University," Liver Transplantation, vol. 11, no. 11, pp. 1332-1342, 2005.

[88] G. Morgan and R. Superina, "Congenital absence of the portal vein: two cases and a proposed classification system for portasystemic vascular anomalies," Journal of Pediatric Surgery, vol. 29, no. 9, pp. 1239-1241, 1994.

[89] G. Acharya, S. Mehra, R. Patel, S. Frunza-Stefan, and H. Kaur, "Fatal nonhepatic hyperammonemia in ICU setting: a rare but serious complication following bariatric surgery," Case Reports in Critical Care, vol. 2016, Article ID 8531591, 6 pages, 2016.

[90] A. Açikalin, N. R. Dişel, E. Ç. Direk, M. T. Ilginel, A. Sebe, and Ş. Biçakçi, "A rare cause of postpartum coma: isolated hyperammonemia due to urea cycle disorder," American Journal of Emergency Medicine, vol. 34, no. 7, pp. 1324.e3-1324.e4, 2016.
[91] M. Alameri, M. Shakra, and T. Alsaadi, "Fatal coma in a young adult due to late-onset urea cycle deficiency presenting with a prolonged seizure: a case report," Journal of Medical Case Reports, vol. 9, no. 1, article 267, 2015.

[92] G. P. Bijvoet, C. J. van der Sijs-Bos, J. P. Wielders, and O. A. Groot, "Fatal hyperammonaemia due to late-onset ornithine transcarbamylase deficiency," The Netherlands Journal of Medicine, vol. 74, no. 1, pp. 36-39, 2016.

[93] O. M. Panlaqui, K. Tran, A. Johns, J. McGill, and H. White, "Acute hyperammonemic encephalopathy in adult onset ornithine transcarbamylase deficiency," Intensive Care Medicine, vol. 34, no. 10, pp. 1922-1924, 2008.

[94] T. Mahmood and K. Nugent, "Nonhepatic hyperammonemic encephalopathy due to undiagnosed urea cycle disorder," Proceedings, vol. 28, no. 3, pp. 375-377, 2015. 


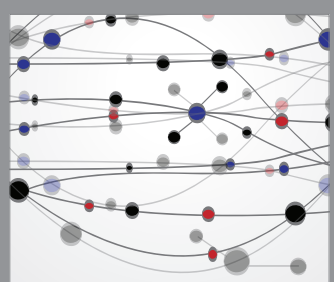

The Scientific World Journal
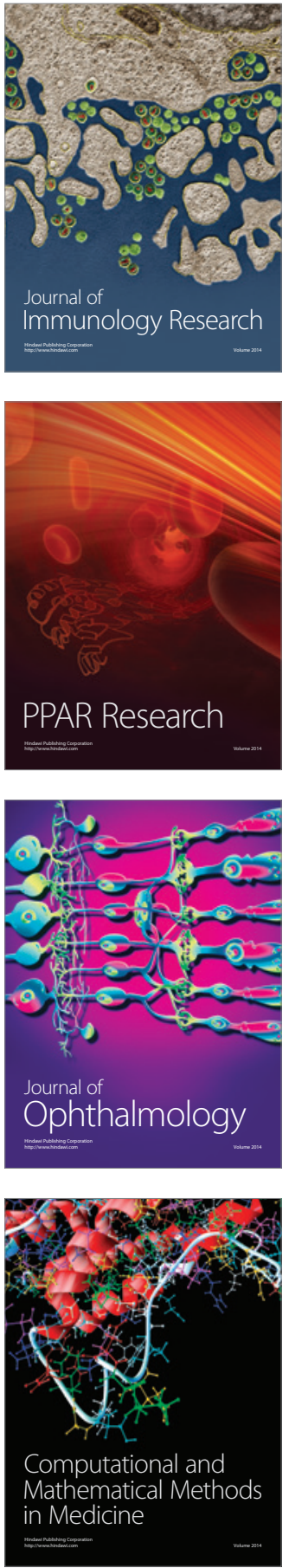

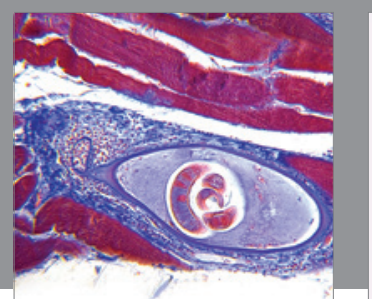

Gastroenterology Research and Practice

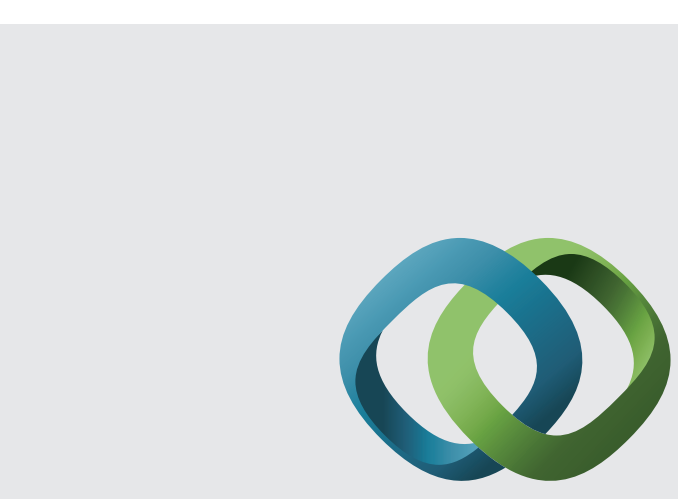

\section{Hindawi}

Submit your manuscripts at

http://www.hindawi.com
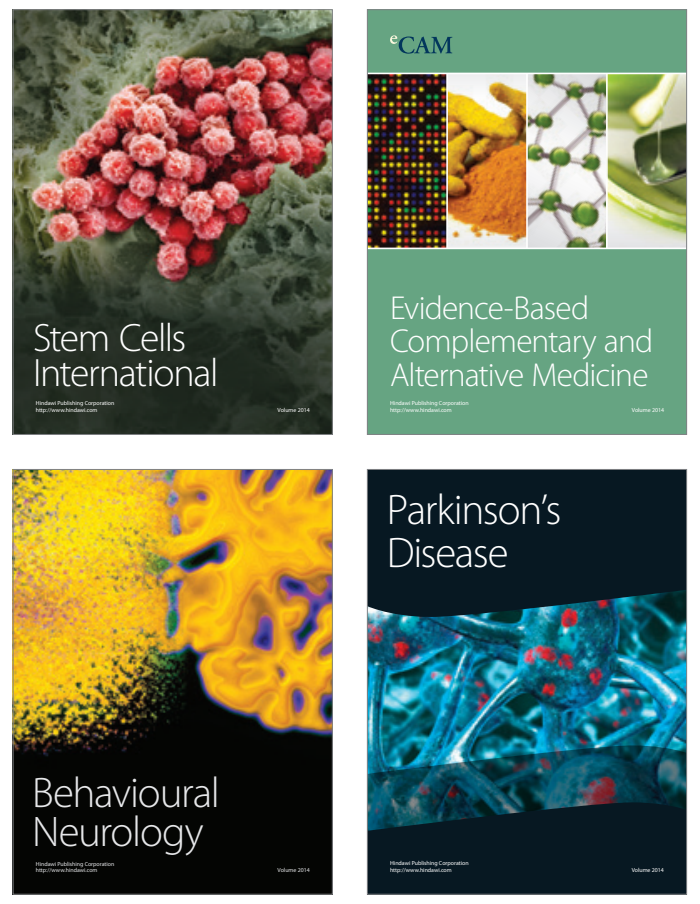
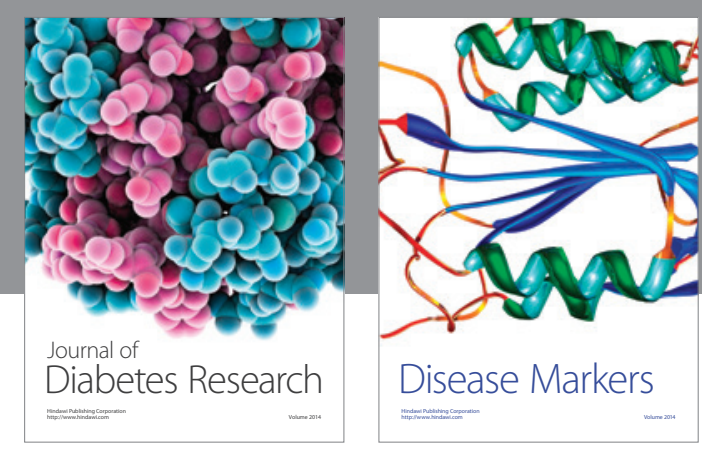

Disease Markers
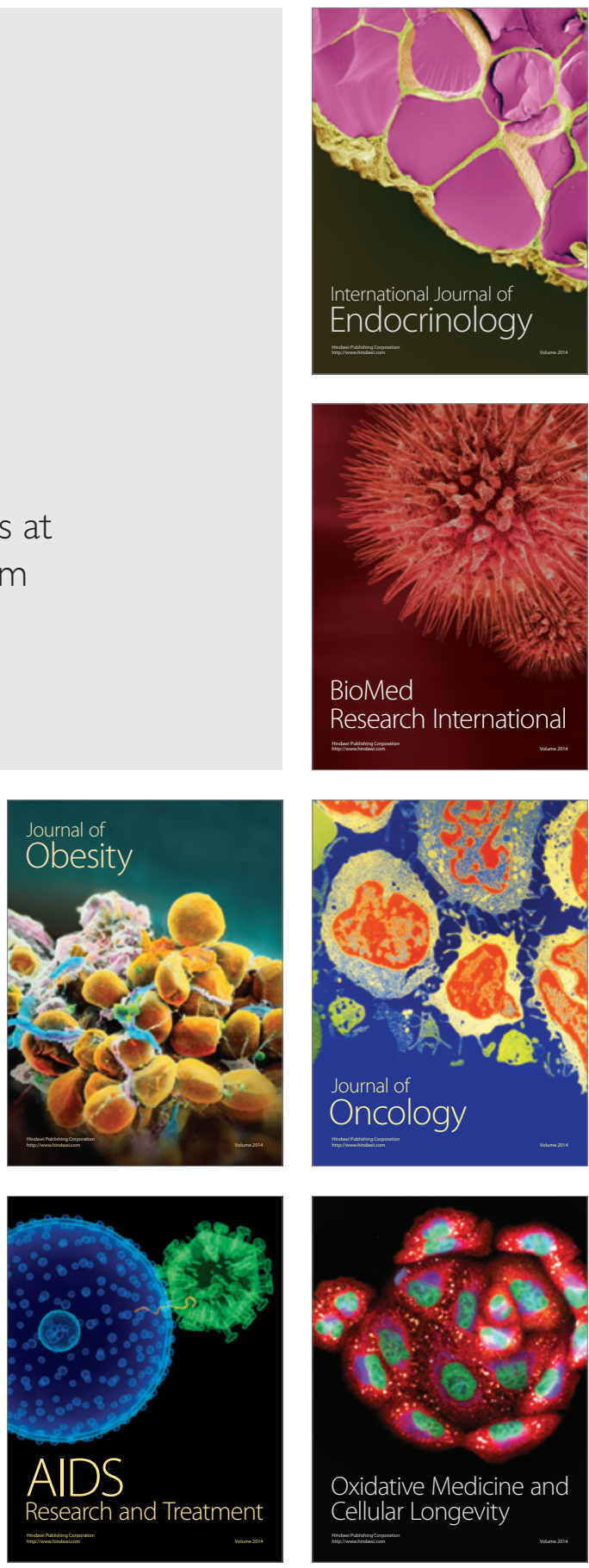